\title{
Informationen und Empfehlungen der Deutschen Gesellschaft für Pneumologie und Beatmungsmedizin e.V. und der Paul-Ehrlich-Gesellschaft für Chemotherapie e.V. zum Ausbruch der Influenza A(H7N9)-Virus-Infektion beim Menschen
}

\author{
Informations and Recommendations of the German Respiratory Society and the Paul-Ehrlich-Society \\ for Chemotherapy Concerning the Outbreak of Influenza A(H7N9) Virus Infections in Humans
}

Autoren

Institute
G. Höffken ${ }^{1}$, P. Kern² ${ }^{\text {, U. Buchholz }}{ }^{3}$, S. Ewig ${ }^{4}$, T. Schaberg ${ }^{5}$

Die Institutsangaben sind am Ende des Beitrags gelistet.
Bibliografie

Dol http://dx.doi.org/

10.1055/s-0033-1344807

Online-Publikation: 23.10.2013

Pneumologie 2013; 67: 599-604

(c) Georg Thieme Verlag KG

Stuttgart · New York

ISSN 0934-8387

\section{Korrespondenzadresse}

Prof. Dr. Gert Höffken

Universitätsklinikum

Carl-Gustav-Carus

Medizinische Klinik I

Bereich Pneumologie

Fetscherstr. 74

01307 Dresden

gert.hoeffken@uniklinikum-

dresden.de

\section{Zusammenfassung \\ $\nabla$}

Seit März 2013 wurden aus der VR China die ersten Fälle einer aviären Influenza-Infektion beim Menschen durch Influenza A(H7N9) der Weltgesundheitsorganisation gemeldet. Es scheint sich bei diesem Virustyp um eine Reassortante, bestehend aus mindestens vier unterschiedlichen aviären Influenzaviren, zu handeln, wobei einzelne Gensegmente human-ähnliche Influenza-Signaturen aufweisen. Bis zum 11. August 2013 wurden 135 Menschen infiziert, von denen 44 (33\%) verstarben. Der klinische Verlauf ist u.a. durch Fieber, Husten, gastrointestinale Symptome, Lympho- und Thrombopenie sowie bei ca. einem Viertel der Fälle durch das rasche Auftreten eines akuten Atemnotsyndroms gekennzeichnet. Jeder Verdachtsfall muss umgehend an das zuständige Gesundheitsamt gemeldet werden. Obwohl eine Mensch-zu-Mensch-Übertragung bisher nichtzweifelsfrei nachgewiesen wurde, sollten strikte Hygienemaßnahmen eingehalten werden. Der Nachweis einer A(H7N9)-Infektion basiert auf der Real-Time-Nukleinsäurekettenreaktion. Schon bei Verdacht auf eine Influenza A(H7N9)-Infektion sollte eine Therapie mit Neuraminidase-Inhibitoren eingeleitet werden, selbst wenn schon mehr als $48 \mathrm{~h}$ seit Symptombeginn vergangen sind. Zum gegenwärtigen Zeitpunkt ist die weitere Entwicklung dieses Ausbruchs von aviären Influenzainfektionen beim Menschen schwer vorherzusagen.

\section{Vorbemerkung}

Diese Stellungnahme und Empfehlung soll die ärztliche Öffentlichkeit in Krankenhäusern und Praxen in Deutschland, im öffentlichen Gesundheitsdienst sowie alle weiteren Akteure im Gesundheitswesen über den Ausbruch einer Infektion durch das Influenzavirus Typ A(H7N9) in China informieren. Die Kenntnis über Ausbrei-

\section{Abstract \\ $\nabla$}

In March 2013, the first cases of avian influenza virus infections in humans were reported by the authorities of the PR of China to the World Health Organization. This influenza A(H7N9) virus comprises genes of at least four different avian influenza viruses, some segments mimicking humanlike influenza-signatures. Until 11 August, 2013 135 humans were infected, 44 (33\%) died. The clinical course is characterized by fever, cough, gastrointestinal symptoms, lympho- and thrombopenia as well by the rapid onset of an acute respiratory distress syndrome in nearly $25 \%$ of the cases. Although human to human transmission may have occurred only in the context of three clusters, strict hygiene measures should be instituted and any suspect case should be reported to the local health authorities immediately. The detection of influenza $\mathrm{A}(\mathrm{H} 7 \mathrm{~N} 9)$ is based on real-time polymerase chain reaction (PCR). Antiviral treatment should be initiated as early as possible for suspect, probable or confirmed cases, even when 48 hours have passed after symptom onset. At present the future development of this epidemic cannot be predicted. tung, klinische Präsentation, Komplikationen und Prognose soll eine angemessene Reaktion und koordinierte Vorgehensweise (Erkennen eines Indexfalles, Isolier- und Hygienemaßnahmen, Diagnostik, Therapie und Meldung an die zuständigen Behörden) sicherstellen, um adäquat auf einen möglichen Erkrankungsfall in Deutschland reagieren zu können. Sie basiert im Wesentlichen auf den Veröffentlichungen des Robert Koch-In- 
stitutes, der Weltgesundheitsorganisation, der amerikanischen Centers for Disease Control and Prevention (CDC), der European Centre for Disease Control and Prevention (ECDC) sowie auf der zu diesem Thema publizierten wissenschaftlichen Literatur.

\section{Einleitung \\ $\nabla$}

Anfang März 2013 wurden die ersten Fälle einer Infektion beim Menschen durch ein Influenza A(H7N9)-Virus in östlichen Provinzen der Volksrepublik China beschrieben. Es handelt sich um ein neues, bisher nicht bei Menschen als Krankheitserreger aufgetretenes Vogelgrippevirus, das in verschiedenen Provinzen im Osten der Volksrepublik China schwere respiratorische Infektionen, zum Teil mit tödlichem Ausgang, hervorgerufen hat [1-3]. Mittlerweile wurden der Welt-Gesundheitsorganisation WHO bis zum 11.8.2013 insgesamt 135 Fälle mit akuter Influenza A (H7N9)-Infektion gemeldet, von denen 44 an der Erkrankung verstorben sind (Todesrate 33\%) [4]. Betroffen sind alle Altersklassen, vorwiegend männliche Personen aus einem urbanen Umfeld. In den meisten Fällen (bis auf einige Familien-Cluster) fand sich epidemiologisch keine Verbindung zwischen den Erkrankten, in der Mehrzahl der Fälle lag ein Kontakt mit Geflügel (Hühner oder Enten) vor [3].

Da sich die Untersuchungen der chinesischen Gesundheitsbehörden auf die schweren Fälle von unteren Atemwegsinfektionen konzentrierten, ist eine realistische Einschätzung der klinischen Manifestation und der wirklichen Letalität (bezogen auf alle Infizierten) zum jetzigen Zeitpunkt nicht möglich. Der hohe Prozentsatz an Kontakten mit lebenden Tieren könnte für eine Übertragung des Virus von augenscheinlich nicht erkrankten Tieren auf den Menschen sprechen. Eine direkte Mensch-zu-Mensch-Übertragung kam im Rahmen von drei Häufungen in Frage, konnte bisher jedoch noch nicht zweifelsfrei festgestellt werden.

Es gibt Fragen zur Epidemiologie, die zur Zeit noch offen bleiben müssen, wie das natürliche Vorkommen der Viren in der Tierwelt, die Haupt-Expositions- und Übertragungswege bei Infektion des Menschen oder die Verbreitung in der tierischen und menschlichen Population.

\section{Aviäre Influenzaviren}

$\nabla$

Das natürliche Reservoir der aviären Influenzaviren sind Vögel, insbesondere Wasservögel, wo das Virus gut angepasst ist und nur selten zu Erkrankungen führt. Bei anderen Spezies, wie Geflügel und Säugern, ist die Pathogenität der Viren abhängig vom Virustyp und Wirt. Neben hochpathogenen aviären Influenzaviren (H5 oder H7), die eine hohe Letalität für infiziertes Geflügel (Hühner und Puten) besitzen, werden niedrigpathogene VogelInfluenzaviren unterschieden, die auch bei H5- und H7-Viren vorkommen können und im Geflügel kaum oder nur geringe Krankheitszeichen hervorrufen. Die Übertragung von aviären Influenzaviren auf den Menschen ist nicht sehr effektiv und wurde zum Beispiel für das Influenza A(H5N1)-Virus in 10 Jahren bisher bei ca. 600 Personen nach (überwiegend) engem Tierkontakt beschrieben, wobei die Letalität mit fast $60 \%$ nach gegenwärtigem Wissenstand offensichtlich höher ist als bei Influenza A(H7N9) [5].

Das neue Influenza A(H7N9)-Virus ist nach molekularbiologischen Untersuchungen eine Mischform (Reassortante) aus mindestens vier unterschiedlichen aviären Influenza-Viren. Für Ge- flügel ist das Virus niedrigpathogen. Damit fehlen die Erkennungszeichen einer möglichen Infektionsgefahr für den Menschen im Umfeld von erkranktem Geflügel. Eine Mutation im Hämagglutinin deutet darauf hin, dass die Viren eine größere Fähigkeit besitzen, über 2,6 Sialinsäure-Rezeptoren (die auch in den oberen Luftwegen beim Menschen vorkommen) Säugetierzellen zu infizieren [6]. Molekulargenetische Untersuchungen zeigen, dass das Oberflächenprotein Hämagglutinin am ehesten den Gensegmenten von aviären A(H7)-Influenzaviren aus domestizierten Enten der Provinz Zhejiang ähnelt [7]. Influenza A(H7)Viren können selten, meist nach direktem Kontakt mit infiziertem Geflügel, zu Infektionen bei Menschen führen, wobei diese meist mild verliefen [8]. Das für die Neuraminidase kodierende $\mathrm{N}$-Gen scheint von aviären Influenzaviren des Typs A(H11N9) zu kommen, die sporadisch aus Wildvögeln in Korea und China isoliert wurden [6], während die übrigen Gensegmente aus aviären A(H9N2)-Viren stammen, die im gesamten asiatischen Raum anzutreffen sind. Bis Ende Mai 2013 hat die Welttiergesundheitsorganisation OIE den Nachweis von A(H7N9) in über 50 Proben von Geflügel oder Umgebungsproben von Geflügelmärkten aus neun Provinzen in China bestätigt (http://www.oie.int/wahis_2/public/wahid.php/Wahidhome/Home) [8]. Weitere Mutationen auf unterschiedlichen Gensegmenten sprechen für eine stärkere Adaptation der Viren an Säugetierzellen. Vorhersagen über genetische Veränderungen, die eine Mensch-zu-Mensch-Übertragung ermöglichen, sind zum jetzigen Zeitpunkt nicht zu treffen. Eine zusätzliche Mutation spricht für das Vorliegen einer Resistenz gegenüber Amantadin, was von klinischer Bedeutung sein kann. Bis auf eine Erkrankung, bei der bei dem isolierten A(H7N9)-Virus eine Mutation nachgewiesen werden konnte, die mit einer In-vitro-Resistenz gegenüber Neuraminidase-Inhibitoren bei einem anderen N9-Virussubtyp assoziiert war [9], gibt es keine Hinweise auf eine Resistenz gegen Neuraminidase-Inhibitoren.

Insgesamt handelt es sich um ein patchwork-virus, was sich aus mindestens vier aviären Influenzavirustypen rekrutiert, wobei auch einzelne Gensegmente human-ähnliche Signaturen aufweisen [10].

\section{Klinik}

$\nabla$

Nach den bisher vorliegenden Berichten bei den schwerer erkrankten Patienten betrug die Zeit zwischen Erkrankungsbeginn und Hospitalisierung im Durchschnitt 4,5 Tage, bis Auftreten eines akuten respiratorischen Distress-Syndroms 8 Tage und 11 Tage bis zum Tod. Das Spektrum des klinischen Erscheinungsbildes einer Influenza A(H7N9)-Infektion ist gegenwärtig unbekannt. Während die klinisch schweren Verläufe gut dokumentiert sind, ist völlig unklar, wie häufig oligo- bzw. asymptomatische Infektionen sind bzw. wie sie sich klinisch äußern. Dies hat erhebliche epidemiologische Bedeutung für die Erkennung und Eindämmung der Infektion.

Mittlerweile liegt eine retrospektive Analyse von 111 Patienten vor, bei denen die Infektion durch das Influenza A(H7N9)-Virus im Labor bestätigt war (110 mittels RT-PCR) [11]. Das Durchschnittsalter lag bei 61 Jahren ( 3 bis 88 Jahren) und unterschied sich damit deutlich vom Median bei der aviären A(H1N1)-Pandemie mit 23 Jahren [12]. Auffällig war, dass 42,3\% der Betroffenen über 65 Jahren alt waren und dass Männer überwogen (Verhältnis 2:1). Diese Unterschiede könnten eventuell Ausdruck einer unterschiedlichen Exposition gegenüber infizierten Tieren auf Märkten und Geflügelfarmen bzw. eines erhöhten Risikos bei 
Vorliegen von Begleiterkrankungen sein, die häufiger in höherem Alter vorkommen. In der Mehrzahl der Fälle (61,3\%) lagen Begleiterkrankungen wie koronare Herzerkrankung, arterielle Hypertonie, Diabetes mellitus und chronisch obstruktive Lungenerkrankung vor. Ein aktiver Raucherstatus war mit einer höheren Letalität assoziiert [13].

Das klinische Bild der Infektion war durch das Auftreten von Fieber (100\%), zum Teil über $39^{\circ} \mathrm{C}$, Husten (90\%) sowie körperliche Schwäche, Luftnot, Hämoptysen und gastrointestinale Symptome (Durchfall oder Erbrechen in 13,5\%) charakterisiert. Paraklinisch imponierte bei der Mehrzahl der Patienten eine Lymphopenie (88\%), Thrombopenie (73\%) sowie bei fast allen Patienten eine Erhöhung der Serumkonzentration von Aspartattransferase, Lactatdehydrogenase und Kreatinkinase. Die Entzündungsmarker C-reaktives Protein und Procalcitonin waren bei 76 bzw. 37\% erhöht. In 108 der 111 (97,3\%) vorliegenden Röntgenfilmaufnahmen des Thorax fanden sich Verschattungen vereinbar mit einer Pneumonie. In einzelnen Fällen wurden Pneumothoraces und Mediastinalemphyseme festgestellt. Nosokomiale Superinfektionen traten bei 5 Patienten (Sepsis) und 18 Patienten (Krankenhaus-erworbene Pneumonie) auf. Die im Sputum oder endotrachealen Aspirat isolierten Erreger waren überwiegend Enterobacteriaceae oder Nonfermenter und entsprachen damit den typischen nosokomialen Pneumonieerregern [14].

85 Patienten wurden auf der Intensivstation behandelt, bei 79 Patienten lag ein akutes Atemnotsyndrom, bei 29 Patienten ein Schockzustand sowie akutes Nierenversagen $(n=18)$ oder eine Rhabdomyolyse $(n=11)$ vor. Eine künstliche Beatmung war bei $82 \%$ der Patienten mit ARDS notwendig, von diesen wurden 20 einer extrakorporalen Membranoxigenierung unterzogen. Prädiktor für das Auftreten eines ARDS war das Vorliegen einer Grunderkrankung, für Tod ausschließlich der Nachweis eines Schocks.

Im Vergleich zur saisonalen Influenzainfektion war die Influenza A(H7N9)-Virus-Infektion durch einen schwereren klinischen Verlauf, vermehrtes Auftreten von Komplikationen, insbesondere Pneumonie und akutes Atemnotsyndrom, gekennzeichnet. Ähnlich wie bei $\mathrm{A}(\mathrm{H} 5 \mathrm{~N} 1)$-Infektionen traten Schnupfen und Halsschmerzen nicht auf, jedoch unterschieden sie sich von Infektionen durch andere A(H7)-Viren durch das Fehlen von Konjunktivitis.

\section{Verdacht}

$\nabla$

Die folgenden Definitionen und Empfehlungen sind der Internetseite des Robert Koch-Institutes (RKI) entnommen (http://www. rki.de/start>Infektionskrankheiten A-Z>Aviäre Influenza). Es sei darauf hingewiesen, dass aktuelle Entwicklungen zur epidemiologischen Situation und neu angepasste Empfehlungen auf dieser Homepage zu entnehmen sind.

Nach Angaben des RKI sollten Ärzte bei folgenden Konstellationen das Vorliegen einer Infektion durch das neue aviäre Influenza A(H7N9)-Virus in Betracht ziehen:

\section{Falldefinition}

- Einzelne importierte Fälle (zum Beispiel durch Reiserückkehrer aus China, die sich dort mit Influenza A(H7N9) infiziert haben) können auch in Deutschland nicht grundsätzlich ausgeschlossen werden. Deshalb sollten Ärzte Patienten, die sich mit einer akuten respiratorischen Symptomatik vorstellen, nach einer Chinareise in den letzten zehn Tagen vor Erkran- kungsbeginn fragen. Vor allem der Aufenthalt in Ostchina in einer der Provinzen, in denen humane Fälle mit Influenza A (H7N9) gemeldet wurden, sollte Anlass zu einer genaueren Reiseanamnese geben [15]. Falls sich die betreffende Person erinnern kann, auf einem „wet market“ (wo lebende Tiere, insbesondere auch Geflügel, verkauft werden) gewesen zu sein, würde dies die deutsche Falldefinition für einen Verdachtsfall auf Influenza A(H7N9) erfüllen, ebenso bei einem vorherigen Kontakt mit einem bestätigten humanen Fall mit Influenza A(H7N9)-Infektion. In diesem Fall sollte die Erkrankung des Patienten labordiagnostisch abgeklärt werden. Verdachtsfälle von humanen Erkrankungen mit aviärer Influenza sind dem Gesundheitsamt nach $\S 1$ der Aviären-InfluenzaMeldepflicht-Verordnung zu melden.

- Ohne erinnerten beziehungsweise bekannten „Kontakt“ zu Vögeln, deren Ausscheidungen sowie rohen oder nicht ausreichend durchgegarten Geflügelprodukten in den betroffenen Gebieten Chinas würde ein Patient mit Reiseanamnese Ostchina in den zehn Tagen vor Erkrankungsbeginn nur dann laut Falldefinition die Kriterien für einen Verdachtsfall erfüllen, wenn die Symptomatik dem in der Falldefinition beschriebenen klinischen „Vollbild“ entspräche, das heißt bei einer schweren akuten respiratorischen Erkrankung wie zum Beispiel einer radiologisch bestätigten Pneumonie (Quelle: Buda, Buchholz, Haas, Dt. Ärzteblatt 2013). Die Falldefinition des RKI zu H7N9 ist abrufbar unter: http://www.rki.de/DE/Content/ InfAZ/A/AviaereInfluenza/Falldefinition_H7N9.html.

- Bestätigter Fall: Person mit labordiagnostischem Nachweis des Influenzavirus A(H7N9).

\section{Schutzmaßnahmen \\ $\nabla$}

Da bei Verdacht auf eine aviäre Influenza A(H7N9)-Infektion eine Mensch-zu-Mensch-Übertragung zum gegenwärtigen Zeitpunkt nicht ausgeschlossen werden kann, ist eine Transmission des Virus durch entsprechende Maßnahmen zu unterbinden. Zum Schutz des Personals sollte eine erweiterte Basishygiene mit zusätzlichem Schutz der Atemwege durchgeführt werden. Hierfür sollten vorzugsweise Atemschutzmasken (FFP1/FFP2) Verwendung finden. Als Behelf kann ein Mund-Nasen-Schutz genutzt werden, dieser schützt jedoch bestimmungsgemäß in erster Linie Mund und Nase vor Spritzern und Berührungen durch kontaminierte Hände. Grundsätzlich ist auf den korrekten Sitz der Maske zu achten. Auch der Patient sollte zur weiteren Erhöhung des Schutzes einen MNS tragen, es sei denn, er toleriert dies nicht.

1. Bezüglich der Schutzmaßnahmen bei Patienten mit einer A(H7N9)-Infektion gilt der Beschluss des Ausschusses für biologische Arbeitsstoffe (ABAS) 609 (http://www.baua.de/de/ Themen-von-A-Z/Biologische-Arbeitsstoffe/TRBA/Beschluss609.html) Der Patient sollte isoliert werden.

2. Die persönliche Schutzausrüstung sollte umfassen:

(a) Schutzbrille, (b) Atemschutz (FFP2 für Pflege, Behandlung, Untersuchung; FFP3 für Aerosol-generierende Maßnahmen, wie Absaugen, Bronchoskopie, Intubation), (c) Schutzkittel, und (d) Einweghandschuhe.

3. Wenn es der Patient toleriert, sollte dieser beim Kontakt mit anderen, nicht infizierten Personen eine Mund-Nasen-Maske aufsetzen.

4. Die CDC empfiehlt für das Management der Versorgungseinheit, auf der A(H7N9)-Patienten betreut werden, eine sorgfältige Kontrolle der Beschäftigten hinsichtlich des Auftretens 
von Krankheitssymptomen, die auf einen respiratorischen Infekt hindeuten könnten. Nähere Erläuterungen sind der Publikation der CDC zu entnehmen [16].

\section{Diagnostik}

Bei Verdacht auf eine Infektion durch Influenza A(H7N9) sollte das Probenmaterial an das Nationale Referenz-Zentrum für Influenza (NRZ) in Berlin (Adresse s. u.) versandt werden. Dabei ist Folgendes zu beachten:

1. Vorab-Information an das NRZ für Influenza über die geplante Probenverschickung

2. Die Proben sind als „Biologischer Stoff, Kategorie B“ der UN-Nr. 3373 zuzuordnen und entsprechend zu kennzeichnen. Der Absender mit Kontaktdaten für Rückfragen ist anzugeben. Als geeignete Untersuchungsmaterialien sind anzusehen:

- Nasen- und/oder Rachenabstrich in Transportmedium für die Virusdiagnostik

- Rachenspülwasser

- Nasopharyngeales Sekret

- Trachealsekret

- Bronchiallavage

Die Materialentnahme sollte von geschultem Personal unter Beachtung der geltenden hygienischen Vorschriften und des $\mathrm{Ar}-$ beitsschutzes gemäß dem Beschluss des Ausschusses für Biologische Arbeitsstoffe 609 erfolgen (Bundesanstalt für Arbeitsschutz und Arbeitsmedizin).

Die Verpackung besteht aus drei Komponenten:

1. Probengefäß (z. B. Monovette) entspricht der Primärverpackung

2. Schutzgefäß als Sekundärverpackung (flüssigkeitsdicht verschraubtes Plastikröhrchen mit saugfähigem Material)

3. Möglichst gefütterter Umschlag aus reißfestem Material oder einer entsprechenden kastenförmigen Verpackung aus Pappe (Umverpackung).

Die verschlossenen Versandstücke werden an das Nationale Referenzzentrum für Influenza (NRZ) am Robert Koch-Institut, Ansprechpartner Dr. B. Schweiger, Dr. M. Wedde, Tel. 030/187542456 oder -2141, Fax 030/18754-2605, versandt.

Das Robert Koch-Institut empfiehlt, bei einem negativen Testergebnis, aber weiter bestehendem hinreichenden Verdacht auf das Vorliegen einer Influenza A(H7N9)-Virus-Infektion, eine Wiederholung der Probenentnahme, möglichst mit Gewinnung respiratorischer Sekrete der unteren Atemwege (z.B. bronchoalveoläre Lavage) mit erneuter PCR-Testung. Ein weiter bestehender Verdacht begründet sich bei positiver Reiseanamnese, ungewöhnlich schwerem klinischem Verlauf (Pneumonie oder ARDS) oder intensiver Exposition.

Darüber hinaus sollte bei allen Patienten mit schweren Pneumonien und akutem Atemnotsyndrom eine sorgfältige virologische Diagnostik mit Berücksichtigung der saisonalen Influenzaviren (Influenza $\mathrm{A}(\mathrm{H} 3 \mathrm{~N} 2)$ ), des pandemischen Influenza $\mathrm{A}(\mathrm{H} 1 \mathrm{~N} 1)$ pdm09, aber auch anderer, zum akuten Atemnotsyndrom führende Erreger, wie MERS-CoV (Middle East Respiratory Syndrome-Coronavirus) erfolgen, wobei bei Ausschluss einer Infektion durch zirkulierende Influenzaviren und fehlender ätiologischer Erklärung des Krankheitsbildes eine weiterführende Charakterisierung von Untersuchungsmaterialien auf Influenza A(H7N9) durch das Nationale Referenzzentrum für Influenza beim RKI zu überlegen ist.
Eine Influenza A(H7N9)-Virus-Infektion gilt als ausgeschlossen, wenn eine repräsentative und verwertbare Untersuchungsprobe mit RT-PCR als negativ bewertet wurde und das Krankheitsbild durch andere Befunde ätiologisch hinreichend erklärt ist bzw. wenn in zwei Serumproben im Abstand von 2 Wochen durch einen spezifischen Test im NRZ keine Antikörper gegen das neue Virus nachgewiesen werden konnten.

\section{Meldung \\ $\nabla$}

Bei Vorliegen einer der Kategorien der Falldefinition erfolgt umgehend eine Meldung gemäß § 1 Aviäre-Influenza-MeldepflichtVerordnung an das zuständige Gesundheitsamt. Dies gilt für niedergelassene und im Krankenhaus tätige Ärzte gleichermaßen. Es empfiehlt sich, das Meldeformular des Robert Koch-Institutes hierfür zu benutzen. Die Meldung erfolgt namentlich mit Angaben des Adressaten und Kontaktdaten. Das Meldeformular ist abrufbar unter http://www.rki.de/DE/Content/Infekt/IfSG/Meldeboegen/Arztmeldungen/Aviare-Influenza_Meldebogen_Vorschlag_des_RKI.html.

\section{Labordiagnostischer Nachweis}

Der Nachweis von Influenza A(H7N9) erfolgt mittels einer RealTime-Nukleinsäureamplifikation, wobei im ersten Schritt über eine generische Matrixgen-Nukleinsäureamplifikation Influenza A und anschließend in einem zweiten Schritt der Subtyp H7Hämagglutinin und N9-Neuraminidase mit spezifischen PCRs (Real Time RT-PCR Protocol for the Detection of Avian Influenza A(H7N9) Virus) eingesetzt wird.

Zu beachten ist, dass Influenza-Schnelltests aufgrund der zur Zeit noch bestehenden Unsicherheit hinsichtlich ihrer diagnostischen Spezifität und Sensitivität bei Influenza A(H7N9) nicht eingesetzt werden sollen. Ein positiver Influenza A-Nachweis könnte allerdings auf eine Influenza A(H7N9)-Virus-Infektion hinweisen.

\section{Klinisches Management und Therapie}

Aufgrund der bisher beschriebenen klinischen Verläufe umfasst das Management von Patienten mit einer Influenza A(H7N9)-Virus-Infektion eine umfassende klinisch-differenzialdiagnostische Aufarbeitung und sorgfältige Überwachung der Patienten zum raschen Erkennen von Komplikationen. Dies beinhaltet die Isolierung des Patienten, bei Bedarf Sauerstoffgabe unter Kontrolle der Blutgasanalyse, ggf. die empirische Gabe von Antibiotika entsprechend den Leitlinien für die schwere ambulant erworbene Pneumonie sowie Prophylaxe thromboembolischer Komplikationen [17]. Bei Vorliegen eines primär schweren Verlaufes bzw. Auftreten von Komplikationen wie akutes Atemnotsyndrom, Sepsis oder Nierenversagen stehen intensivmedizinische Maßnahmen wie nichtinvasive Beatmung, invasive Beatmung, extrakorporale Membranoxigenierung (ECMO), Flüssigkeitsmanagement und Vasopressorgabe im Vordergrund.

Der Stellenwert einer Behandlung mit Neuraminidase-Inhibitoren ist zum gegenwärtigen Zeitpunkt schwer zu bestimmen. Allerdings zeigen In-vitro-Untersuchungen, dass Oseltamivir und Zanamivir die Replikation des Influenza A(H7N9)-Virus zu hemmen vermag [18]. 
In Anbetracht fehlender Alternativen wird daher bei allen Formen einer Infektion die unmittelbare Einleitung einer Behandlung mit Oseltamivir (Tamiflu ${ }^{\odot}$ ) angeraten. Die empfohlene Dosierung bei Erwachsenen beträgt $75 \mathrm{mg}$ zweimal täglich bei Nierengesunden. In den bisher vorliegenden Veröffentlichungen werden Tages-Dosierungen von $150 \mathrm{mg}$ bis $300 \mathrm{mg}$ Oseltamivir angegeben, die damit laut Fachinformation jenseits der für Deutschland zugelassenen Dosis liegen [19]. In der Publikation von Gao et al. führte die Verabreichung von Oseltamivir in den beschriebenen Dosen innerhalb von 6 Tagen nach Beginn der Therapie zu einer Negativierung der respiratorischen Proben mittels RT-PCR, Angaben zu Patienten ohne Behandlung mit Neuraminidasehemmern fehlen allerdings [11]. Das in Oseltamivir-Kapseln enthaltene Pulver kann in einer Suspension aufgenommen werden (siehe Fachinformation) und bei beatmeten Patienten über eine Magensonde appliziert werden, allerdings sollte beachtet werden, dass infolge einer verzögerten Magenentleerung eine ausreichende Absorption fraglich ist (http://www. who.int/influenza/resources/documents/ClinicalManagement07. pdf).

Daten zum klinischen Einsatz von Zanamivir (Relenza ${ }^{\odot}$ ) stehen bisher für A(H7N9)-Infektionen nicht zur Verfügung; der Wirkstoff, der inhalativ verabreicht werden muss, ist prinzipiell aber genauso wirksam wie Oseltamivir. Er ist insbesondere bei Patienten, bei denen keine enterale Applikation möglich ist, eine Alternative. Zanamivir ist in der intravenösen Form in Deutschland nicht im Handel, er kann bei der Herstellerfirma GlaxoSmithKline GmbH \& Co KG/München angefragt werden (Details zum Compassionate Use von Relenza unter Tel.: Tel. 0800 1011744). Es läge damit ein Heilversuch vor, der die Aufklärung und Zustimmung des Patienten bzw. seines gesetzlichen Vertreters erfordert. Die Zanamivir-Dosis in der zugelassenen Form als Pulverinhalation beträgt bei Erwachsenen $2 \times 2$ à $5 \mathrm{mg}$ pro Tag [20].

\section{Empfehlung}

Aufgrund der gut dokumentierten Wirksamkeit der Neuraminidase-Inhibitoren bei Infektionen durch saisonale und pandemische Influenzaviren sowie bei A(H5N1)-Virus-Infektionen und infolge der hohen Rate an sekundären Komplikationen und der hohen Sterblichkeit bei einer A(H7N9)-Virus-Infektion wird empfohlen, alle Verdachtsfälle und nachgewiesenen Fälle antiviral mit Neuraminidase-Inhibitoren zu behandeln [21,22].

- Als Verdachtsfall gilt eine Influenza-ähnliche Erkrankung (ILI) bei einem Patienten aus dem Risikogebiet in China (s. RKI-Falldefinition).

- Diese Empfehlung gilt für alle Fälle, unabhängig vom Vorliegen der klassischen Risikofaktoren für einen schweren Verlauf, wie Alter unter 2 Jahre oder über 64 Jahre, Schwangerschaft bzw. Patienten mit chronischen Grunderkrankungen wie COPD, zerebrovaskuläre oder kardiale Erkrankungen.

- Die virologische Diagnostik und der Beginn der antiviralen Therapie sollten parallel erfolgen, die Diagnostik darf den Beginn der Therapie nicht verzögern.

- Die Therapie sollte so früh wie möglich begonnen werden, unabhängig vom Beginn der klinischen Symptomatik (eine Begrenzung der Therapieindikation auf die ersten $48 \mathrm{~h}$ nach Symptomenbeginn besteht nicht); diese Empfehlung berücksichtigt die potenziell tödlichen Verläufe, die Erfahrung mit humanen A(H5N1)-Infektionen, die auf eine Wirksamkeit von Neuraminidasehemmern hinweisen [23], und die Empfehlun- gen der CDC (http://www.cdc.gov/flu/avianflu/h7n9-antiviraltreatment.htm) sowie der WHO (http://www.who.int/ influenza/human_animal_interface/influenza_h7n9/ WHO_H7N9_review_31May13.pdf).

- Die Therapiedauer beträgt bei unkomplizierten Verläufen 5 Tage.

- Bei komplizierten Verläufen wird die ausschließliche Verabreichung von Oseltamivir oral empfohlen, da die Wirksamkeit von inhaliertem Zanamivir unsicher ist.

- Die Therapiedauer ist bei komplizierten und schweren Verläufen unklar. Eine Verlängerung der Behandlung bis auf 10 Tage sollte sich nach klinischen Parametern und virologischen Kriterien (Verlaufsuntersuchungen der Viruslast in respiratorischen Sekreten mittels RT-PCT) orientieren.

- Bei einem geplanten Compassionate use von Zanamivir intravenös sollte die Herstellerfirma kontaktiert werden (s. o.).

- In Einzelfällen sollte auch eine Erhöhung der Dosis über den zugelassenen Dosisbereich hinaus erwogen werden. So wurden Dosierungen bis zweimal 150 mg Oseltamivir pro Tag bei normaler Nierenfunktion und sehr schweren Verläufen beschrieben [24]. Dies ist in jedem Fall als Individualentscheidung zu protokollieren.

\section{Ausblick \\ $\nabla$}

Zum gegenwärtigen Zeitpunkt ist die weitere Entwicklung des bisher noch auf China begrenzten Ausbruchs einer aviären Influenzainfektion bei Menschen schwer vorherzusagen. Nach übereinstimmenden Einschätzungen der WHO und der ECDC sind die momentanen Risiken als gering einzuschätzen. Man sollte allerdings berücksichtigen, dass von drei wesentlichen Voraussetzungen für eine Pandemie zwei durch das neue Influenza A (H7N9)-Virus erfüllt werden [25]: 1. Fehlende Immunität in der menschlichen Population und 2. ausreichende Pathogenität und Virulenz der Viren mit Adaptation an Säugetierzellen durch Mutationen, die zu einer verbesserten Effektivität der Replikation in Säugetierzellen führen. Die für die Entwicklung einer Pandemie wichtigste Eigenschaft eines Virus ist die fortgesetzte Menschzu-Mensch-Übertragung. Im Rahmen von drei kleinen Häufungen ist es möglicherweise bereits zu Mensch-zu-Mensch-Übertragungen gekommen [3,22], nicht jedoch zu anhaltenden Infektketten. Es ist unklar, wie rasch die Influenza A(H7N9)-Viren die Fähigkeit entwickeln, sich an den menschlichen Organismus anzupassen [26]. Würde dies eintreten, hätte dies erhebliche epidemiologische Konsequenzen.

Die Aufgabe besteht daher darin, die ärztliche Öffentlichkeit adäquat zu informieren, um möglicherweise auftretende (importierte oder bereits von diesen angesteckte) Fälle frühzeitig zu erkennen und zu behandeln. Eine Meldung an das örtliche Gesundheitsamt ermöglicht eine umgehende Fall- und Kontaktpersonenuntersuchung.

\section{Interessenkonflikt}

$\nabla$

Die Autoren geben an, dass kein Interessenkonflikt besteht. 
Institute

${ }^{1}$ Universitätsklinikum Dresden, Fachkrankenhaus Coswig

2 Universitätsklinikum Ulm

${ }^{3}$ Robert Koch-Institut, Abteilung für Infektionsepidemiologie, Berlin

4 Thoraxzentrum Ruhrgebiet, Herne und Bochum

${ }^{5}$ Zentrum für Pneumologie - Agaplesion Diakonieklinikum Rotenburg

\section{Literatur}

1 World Health Organization. http://www.who.int/influenza/human_ animal_interface/influenza_h7n9/WHO_H7N9_review_31May13.pdf

2 World Health Organization. Number of confirmed cases of avian influenza $\mathrm{A}(\mathrm{H} 7 \mathrm{~N} 9)$ reported to WHO. http://www.who.int/influenza/human_animal_interface/influenza_h7n9/05_Report-webH7N9Number. pdf

3 Li Q Zhou L, Zhou M et al. Preliminary report: epidemiology of the avian influenza A (H7N9) outbreak in China. New Engl J Med 2013 Apr 24 [Epub ahead of print]. DOI: 10.1056/NEJMoa1304617

4 http://www.who.int/csr/don/2013_08_11/en/index.html

5 Koopmans $M$, Wilbrink B, Conyn $M$ et al. Transmission of H7N7 avian influenza A virus to human beings during a large outbreak in commercial poultry farms in the Netherlands. Lancet 2004; 363: 587 - 593

6 Liu D, Shi W, Shi Yet al. Origin and diversity of novel avian influenza A H7N9 viruses causing human infection: phylogenetic, structural, and coalescent analyses. Lancet 2013; 381: 1926-1932

7 Chen Y, Liang W, Yang S et al. Human infections with the emerging avian influenza A H7N9 virus from wet market poultry: clinical and characterisation of viral genome. Lancet 2013; 381: 1916-1925

8 MacKay J. OIE (World Organisation for Animal Health) Statement on Live Market and H7N9. 19. 05. 2013: http://h7n9flunews.com/whoh7n9-report-highlights-the-knowledge-gaps/

9 Uyeki TM, Cox NJ. Global concerns regarding novel Influenza A (H7N9) virus infections. New Engl J Med 2013; 368: 1862 - 1864

10 Liu $Q$, Lu L, Sun Z et al. Genomic signature and protein sequence analysis of a novel influenza A (H7N9) virus that causes an outbreak in humans in China. Microbes Infect 2013; 15: 432-439

$11 \mathrm{Gao} H-N, \mathrm{Lu} \mathrm{H}-\mathrm{Z}$, Cao B et al. Clinical findings in 111 cases of influenza A (H7N9) virus infections. New Engl J Med 2013; 368: 2277- 2285

12 Yang SG, Cao B, Liang RL et al. Antiviral therapy and outcomes of patients with pneumonia caused by influenza A pandemic (H1N1) virus. PLoS One 2012; 7: 01e29652 Epub 2012 Jan 20. DOI: 10.1371/journal. pone.0029652

13 H7N9 deceased mostly smokers. http://www.macaodaily.com/html/ 2013-04/07/content_792500.htm (accessed April 7, 2013)

14 Dalhoff K, Abele-Horn M, Andreas $S$ et al. Epidemiologie, Diagnostik und Therapie erwachsener Patienten mit nosokomialer Pneumonie. Pneumologie 2012; 66: 707 - 765

15 European Center for Disease Control and Prevention (ECDC). Rapid risk assessment: severe respiratory disease associated with a novel influ- enza A virus, A (H7N9) China. Technical reports. ECDC. Updated Risk Assessment 12-04-2013. http://www.ecdc.europa.eu/en/press/news/ Lists/News/ECDC_DispForm.aspx http://www.ecdc.europa.eu/en/publications/Publications/AH7N9.China-rapid-risk-assessment.pdf 3. April 2013

16 Centers for Disease Control and Prevention. Interim guidance for infection control within healthcare settings when caring for patients with confirmed, probable, or cases under investigation of avian Influenza $A(H 7 N 9)$ virus infection. 24.07. 2013: http://www.cdc.gov/flu/ avianflu/h7n9-infection-control.htm

17 Höffken G, Lorenz J, Kern W et al. S3-Leitlinie der Paul-Ehrlich-Gesellschaft für Chemotherapie, der Deutschen Gesellschaft für Pneumologie und Beatmungsmedizin, der Deutschen Gesellschaft für Infektiologie und vom Kompetenznetzwerk CAPNETZ zu Epidemiologie, Diagnostik, antimikrobieller Therapie und Management von erwachsenen Patienten mit ambulant erworbenen unteren Atemwegsinfektionen (akute Bronchitis, akute Exazerbation einer chronischen Bronchitis, Influenza und andere respiratorische Virusinfektionen) sowie ambulant erworbener Pneumonie. Pneumologie 2009; 63: 549-577 und 63: 613-652

18 Yang FF, Wang JL, Jiang $L$ et al. A fatal case caused by novel H7N9 avian influenza a virus in China. Emerg Microbes and Infections 2013; 2: e19 published online 10 April 2013. DOI: 10.1038/emi.2013.22

19 Fachinformation Tamiflu. Grenzach/Wyhlen: Roche;

20 Fachinformation Relenza. München: GlaxoSmithKline;

21 Centers for Disease Control and Prevention. Interim guidance on the use of antiviral agents for treatment of human infections with avian Influenza $\mathrm{A}(\mathrm{H} 7 \mathrm{~N} 9)$. http:www.cdc.gov/flu/avianflu/h7n9-antiviraltreatment.htm

22 World Health Organization. Overview of the emergence and characteristics of avian influenza $\mathrm{A}(\mathrm{H} 7 \mathrm{~N} 9)$ virus.WHO.org

23 Abdel-Ghafar AN, Chotpitayasunondh T, Gao Z et al. Writing Committee of the Second World Health Organization Consultation on Clinical Aspects of Human Infection with Avian Influenza A (H5N1) Virus. Update on avian influenza A (H5N1) virus infection in humans. N Engl J Med 2008; 358: $261-273$

24 Giraud C, Manceau S, Oualha M et al. High levels and safety of oseltamivir carboxylate plasma concentrations after nasogastric administration in critically ill children in a pediatric intensive care unit. Antim Ag Chemother 2011; 55: 433-435

25 Pavia AT. Influenza A(H7N9): From Anxiety to Preparedness. Ann Intern Med 2013 May 14 [Epub ahead of print]. DOI: 10.7326/00034819-159-3-201308060-00653

$26 \mathrm{Qu}$ X, Quian Y-H, Bao C-J et al. Probable person to person transmission of novel avian influenza A(H7N9) virus in Eastern China, 2013: Epidemiological investigation. BMJ 2013; 347: (Published 6 August 2013) DOI: $10.1136 /$ bmj.f4752 